\title{
Normalizing the CPT for Overburden Stress
}

\author{
R. E. S. Moss ${ }^{1}$; R. B. Seed ${ }^{2}$; and R. S. Olsen ${ }^{3}$
}

\begin{abstract}
Effective overburden stress can have a significant influence on cone penetration test (CPT) measurements. This influence can lead to an incorrect assessment of soil strength/resistance for such purposes as liquefaction triggering analysis. For an accurate measurement of tip and sleeve resistance, unbiased by overburden stress, it is essential to normalize these index measurements appropriately. Presented herein is a comprehensive study reviewing all aspects of CPT normalization. A result of this study is a variable normalization procedure for the CPT that is based on both empirical results and theoretical analysis. This paper presents these results in the form of an improved normalization scheme and discusses its application in practice.
\end{abstract}

CE Database subject headings: Cone penetration; Stress; Measurement.

\section{Introduction}

Raw cone penetration test (CPT) measurements can be misleading if overburden stress effects are not properly taken into account. Low overburden stresses, found at shallow soil depths, will result in a reduced measured tip and sleeve resistance, whereas high overburden stresses found at greater soil depths, will result in a logarithmically pronounced increase in measured tip and sleeve resistance.

Overburden stress has a tendency to influence different soils differently. Cohesive soils respond to overburden stress primarily as a function of over consolidation ratio (OCR) and undrained strength $\left(s_{u}\right)$. Cohesionless soils respond to overburden stress primarily as a function of relative density $\left(D_{r}\right)$ and the coefficient of lateral earth pressure $\left(K_{0}\right)$, and to a lesser degree as a function of the angularity, compressibility, and crushing strength of the grains.

The effects of overburden stress on CPT measurements are nonlinear, showing a curve-linear decrease with linear increase in stress. To account for the effects of overburden stress, tip and sleeve resistance values are normalized to a reference stress value. The reference stress value that is convenient, and commonly used in practice, is $1 \mathrm{~atm}$ ( $1 \mathrm{~atm}=101.325 \mathrm{kPa}$ $\left.=1.033 \mathrm{~kg} / \mathrm{cm}^{2}=14.696 \mathrm{psi}=1.058 \mathrm{tsf}\right)$. One atm is also a reasonable reference value because it is a median depth for most geotechnical engineering problems.

\footnotetext{
${ }^{1}$ Assistant Professor, Dept. of Civil and Environmental Engineering, California Polytechnic State Univ., San Luis Obispo, CA 93407 (corresponding author). E-mail: rmoss@calpoly.edu

${ }^{2}$ Professor, Dept. of Civil and Environmental Engineering, Univ. of California at Berkeley, Berkeley, CA 94720.

${ }^{3}$ Researcher, US Army Engineer Research and Development Center (ERDC), Waterways Experiment Station (WES), Vicksburg, MS 39108.
}

Normalization procedures are used routinely in liquefaction studies, which was the impetus for this study. Development of an improved CPT-based liquefaction triggering correlation (Moss et al. 2005) required that an improved normalization scheme be developed to remove the bias imposed by improper overburden stress normalization. Prior liquefaction triggering correlations used a simplified normalization procedure, as described in Youd et al. (2001), that normalized for the average effect of overburden stress on soils types considered liquefiable.

\section{Previous Research}

The bulk of the research on CPT normalization was conducted by Olson (1994), Olson and Malone (1988), Olson and Koester 1995, and Olson and Mitchell 1995. Olsen (1994) used a technique of defining the normalization for tip and sleeve resistance of various soil types based on field and laboratory data. The resistance was measured over a broad range of overburden stresses for a given "uniform" soil strata, and the results plotted as a function of overburden stress in log-log space, giving a linear relationship. The stress normalization exponent for that particular soil state was then the slope of the linear fit, or power law equation, in $\log$-log space (with the symbol $c$ for tip exponent and $s$ for sleeve exponent). This procedure was applied to all soil types where reasonable data existed, which led to the Olsen and Mitchell (1995) chart of normalization exponent contours (Fig. 1). These exponent contours were for normalizing the tip and sleeve resistance as shown below

$$
q_{c, 1}=C_{q} \cdot q_{c}
$$

and

$$
f_{s, 1}=C_{f} \cdot f_{s}
$$

where

$$
C_{q}=\left(\frac{P_{a}}{\sigma_{v}^{\prime}}\right)^{c}
$$




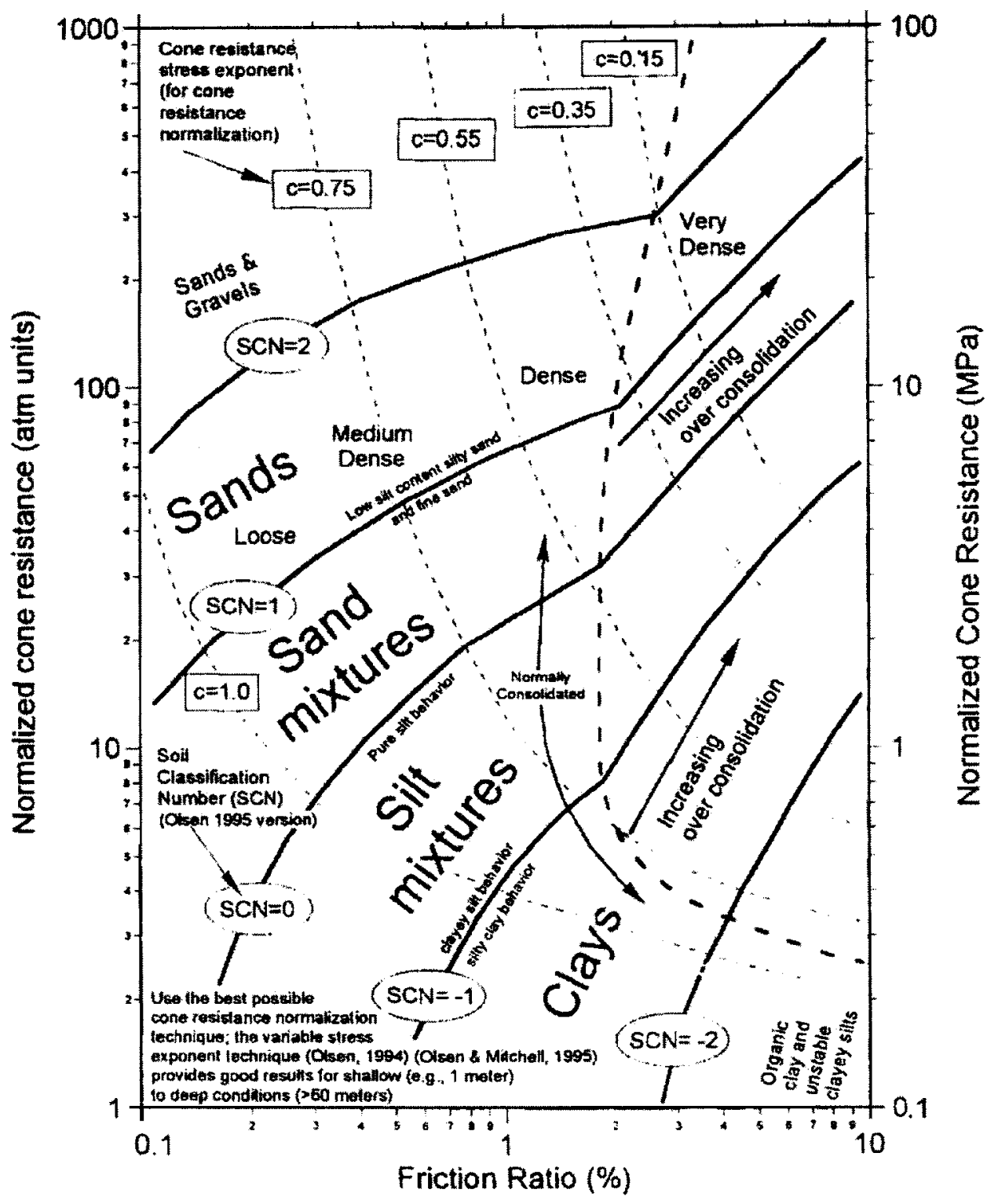

Fig. 1. Variable CPT normalization from Olsen and Mitchell (1995)

$$
C_{f}=\left(\frac{P_{a}}{\sigma_{v}^{\prime}}\right)^{s}
$$

In Eq. (1) $q_{c}=$ raw tip resistance; $f_{s}=$ raw sleeve resistance; $P_{a}=$ atmospheric pressure in compatible units; $\sigma_{v}^{\prime}=$ vertical effective overburden stress; $c$ and $s=$ tip and sleeve normalization exponents; $C_{q}$ and $C_{f}=$ normalizing factors; and $q_{c, l}$ and $f_{s, 1}=$ normalized tip and sleeve resistances. Friction ratio is the percent ratio of sleeve to tip resistance in compatible units, $R_{f}=\left(f_{s} / q_{c}\right) \cdot 100$.

Olsen's work incorporated over 2 decades of field data and an extensive database of chamber test studies by other researchers to deduce the tip normalization for a number of different soil types. Olsen produced a strong basis for cone normalization, and subsequent researchers (e.g., Robertson and Wride 1998) referred to this body of work when addressing normalization.

A limitation of normalization based on empirical data is that a soil layer must be "uniform" and extend over a sufficient depth to be of use in calculating the normalization exponent. Normalization data in sands are generally restricted to chamber test results because of the inherent variability of in situ deposits due to the typical depositional environment associated with this type of soil. For clays, field tests are the only option because of the difficulty of performing accurate chamber studies with this type of soil. For soils other than sands and clays such as silts, high silt content sands, clayey sands, etc., it is difficult if not impossible to find "uniform" layers in the field or to produce quality chamber test specimens to generate normalization data.

Olsen (1994) used a database of chamber data and "uniform" layer field data to populate exponent regions on the characterization chart. The "uniform" layer field data, as shown in Fig. 2, were collected from public and private sources as listed in Olsen (1994). The calibration chamber data, shown in Figs. 3 and 4, came from laboratory studies performed by various researchers, and a compilation and complete list of references of these studies can be found in Salgado-Rodrigues (1993). Olsen's characterization chart (Fig. 1) shows variable tip normalization exponents for different soil types and stress conditions. The sleeve normalization exponent is assumed equal to tip normalization exponent $(s=c)$. 


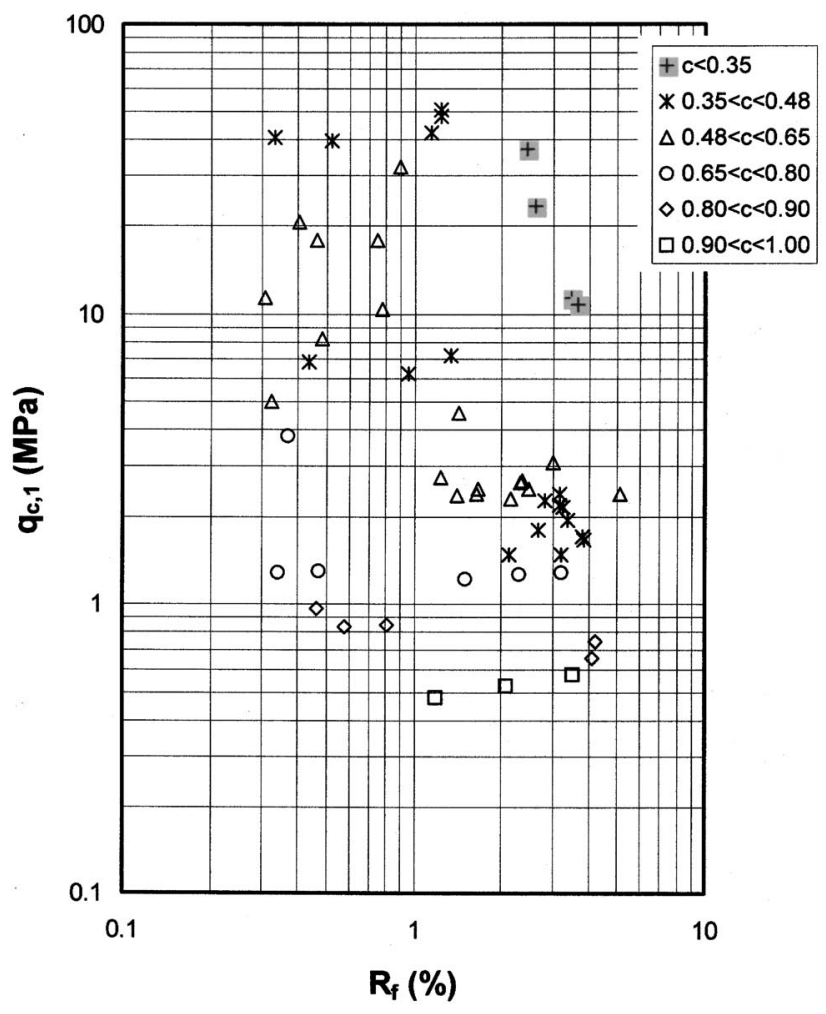

Fig. 2. Tip normalization exponent results from field data (after Olsen 1994)

\section{Theoretical Foundation for Normalization}

To expand on Olsen's work a new approach was taken. This approach was to explore the theoretical foundation of CPT normalization. A thorough review of methods that theoretically predict CPT measurements from fundamental soil properties was performed. There are many methods that have been proposed, including bearing capacity, cavity expansion, strain path, steady state, incremental finite element, and discrete element.

Based on the literature (Keaveny 1985; Keaveny and Mitchell 1988; Mayne 1991; Yu and Houlsby 1991; Salgado-Rodrigues 1993; Collins et al. 1994; Huang and Ma, 1994; Salgado et al. 1997b; Yu and Mitchell 1998; Yu 2000) cavity expansion methods are the most advanced in the application of theoretical CPT tip predictions at depth. Yu and Mitchell (1998), in particular, looked at all theoretical methods that were comparable at the time and found cavity expansion to be the most developed as well as providing the greatest accuracy in CPT predictions over a wide stress range. Bearing capacity methods require ground surface expression of the failed soil, are only valid for very shallow or low overburden stress regimes, and provide a linear approximation to a nonlinear response. Other methods such as steady state, discrete element, strain path, and incremental finite element are promising methods but are not as developed as cavity expansion methods and may currently only predict CPT tip resistance for limited soil and/or stress conditions. Steady state methods were used in this study for qualitative support of the quantitative cavity expansion results. Bishop et al. (1945) were the first to see the analogy between the expansion of a cavity and the penetration of a cone in an elastic medium. Subsequent researchers developed this concept further by incorporating higher order stress-strain relationships to model sands and clays with increasing rigor and

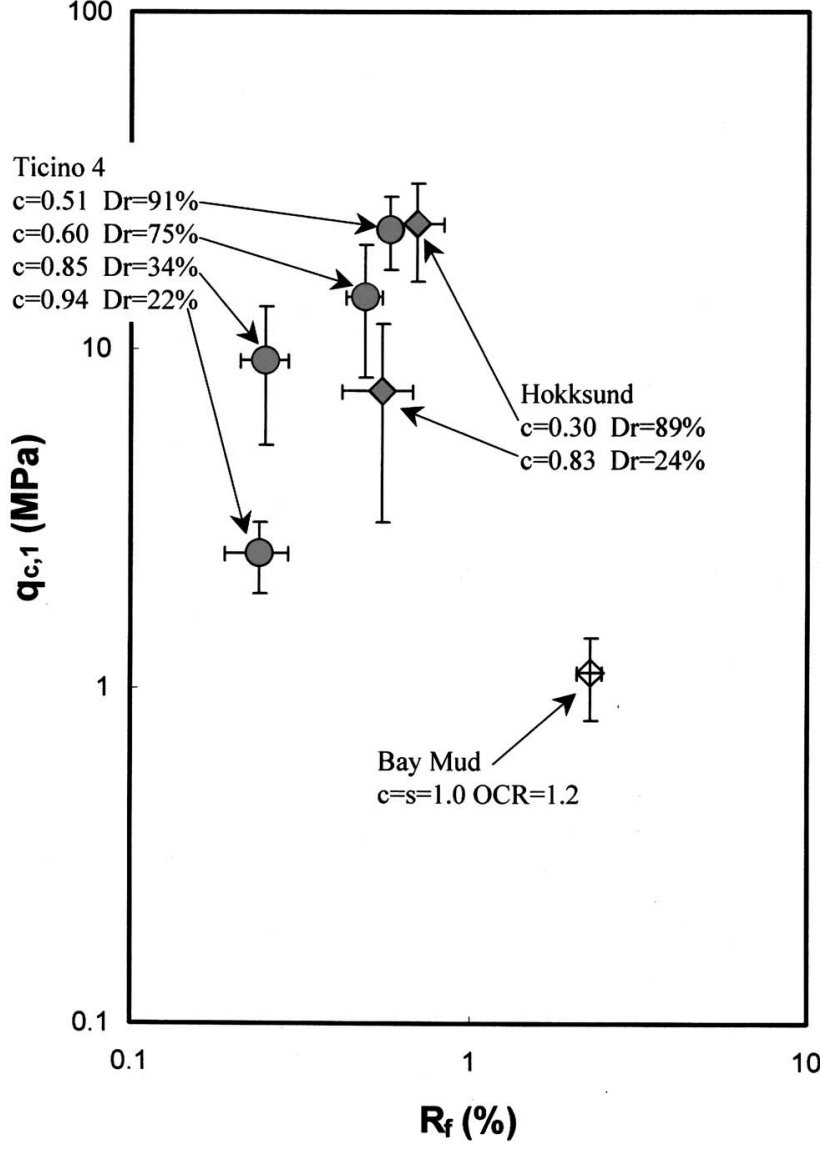

Fig. 3. Tip normalization exponent results from 57 calibration chamber tests and bay mud field data (after Olsen 1994). Data were binned by relative density, with mean relative density shown. Mean and variance of each bin indicated by symbol and whiskers.

accuracy (Vesic 1972; Ladanyi and Johnston, 1974; Baligh 1976; Carter et al. 1986; Yu and Houlsby 1991; Collins et al. 1992; Salgado et al. 1997b).

Cavity expansion, as applied in this study, is a two-step process: (1) a theoretical (analytical or numerical) cavity limit pressure solution is calculated, and (2) this limit pressure is then related to the cone tip resistance. The research described herein uses various cavity expansion solutions to estimate normalization exponents. Because of the complexity of soil behavior and the different solutions required for different types of soil behavior, the discussion of theoretical methods is divided into four soil-state categories: cohesive normally consolidated, cohesive overconsolidated, cohesionless contractive, and cohesionless dilatant.

\section{Cohesive Normally Consolidated (Normally Consolidated Clay)}

Yu and Houlsby (1991) derived an analytical solution for a total stress cylindrical cavity expansion model in normally consolidated cohesive clay. The soil is modeled as a linear elasticperfectly plastic material using Mohr-Coulomb yield criteria. The closed form solution for a standard $60^{\circ}$ cone, with a perfectly rough surface is 


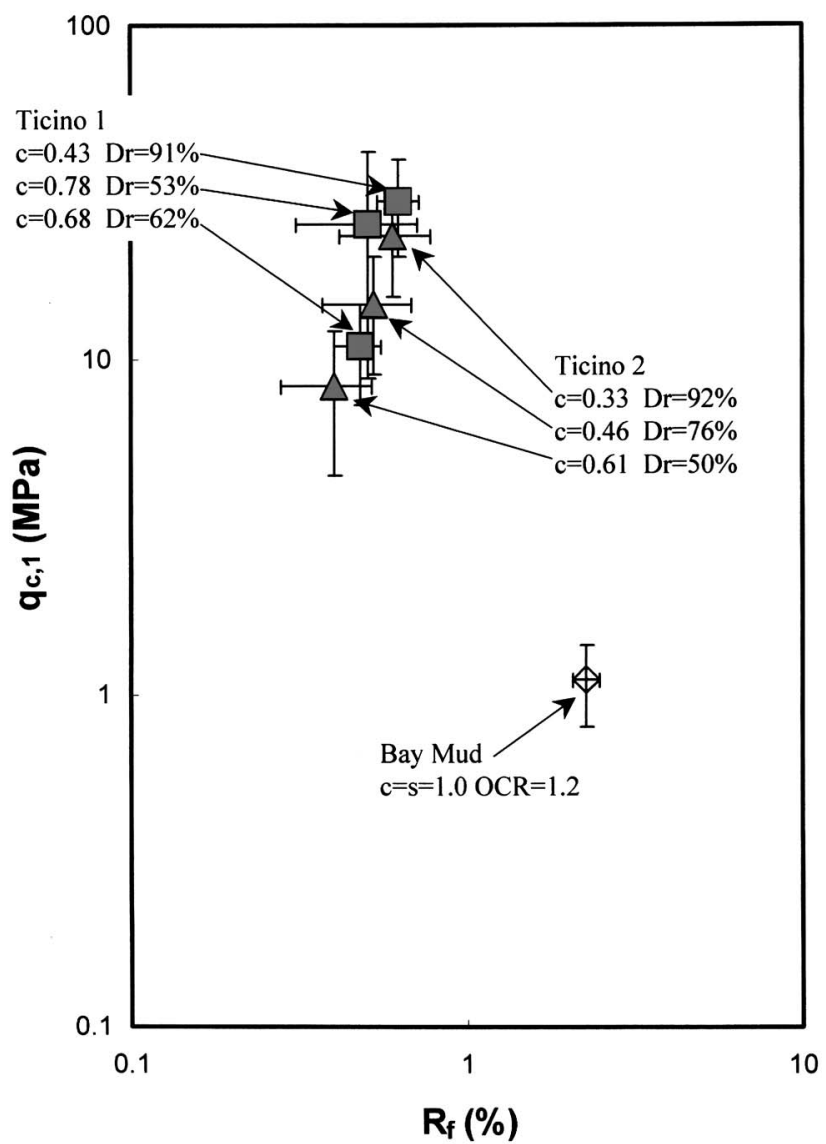

Fig. 4. Tip normalization exponent results from 25 calibration chamber tests and bay mud field data (after Olsen 1994). Data were binned by relative density, with mean relative density shown. Mean and variance of each bin indicated by symbol and whiskers.

$$
q_{c}=N_{c} \cdot s_{u}+\sigma_{m}
$$

where

$$
N_{c}=9.4+1.155 \cdot \ln \frac{\sqrt{3}}{2} \frac{G}{s_{u}}
$$

In Eq. (2) $q_{c}=$ tip resistance; $N_{c}=$ cone factor; $s_{u}=$ undrained strength; $\sigma_{m}=$ mean total stress; and $G=$ elastic shear modulus. Yu and Mitchell (1998) showed that this solution gave good results when compared to empirical data and other analytical solutions.

For this study, Yu's closed form solution [Eq. (2)] was calibrated using chamber data from Kurup et al. (1994). The elastic shear modulus, $G$, was calculated using the equation for $G_{\max }$ from Hardin (1978) and a reasonable value of shear strain. This solution was then used to predict tip resistance, for a CL/CH model clay soil $(\mathrm{OCR}=1.0)$ with a variety of stiffness indices $\left(G / G_{\max }\right)$ under different overburden stresses. The normalization exponent from the results was $c \cong 1$.0.

Currently there is no analytical solution for sleeve resistance. In theory sleeve resistance, $f_{s}$, is a function of $\sigma_{h}^{\prime}$ the effective horizontal pressure and $\delta_{r}$ the effective remolded strength;

$$
f_{s} \propto \sigma_{h}^{\prime} \cdot \tan \delta_{r}
$$

The effective horizontal pressure can be considered a cylindrical cavity expansion limit pressure for sleeve resistance measurements (Keaveny 1985; Keaveny and Mitchell, 1988; Masood,
1990; Masood and Mitchell, 1993). Kurup et al. (1994) chamber data were used to correlate tip and sleeve resistance through a form of Eq. (3). This was then used to approximate the corresponding sleeve resistance. The normalization exponent range for this soil state (cohesive normally consolidated) is shown in the lower right corner of Fig. 5.

Using the steady state method, Yu et al. (2000) showed that tip resistance and friction ratio have similar trends as a function of stiffness index, which is itself a function of overburden stress. Showing that both tip and sleeve resistance can be derived from cylindrical cavity expansion methods, both are a function of stiffness index, and normalization being a function of the cone's response to overburden stress, then it is hypothesized that the tip and sleeve are normalized equivalently for cohesive normally consolidated soils.

\section{Cohesive Overconsolidated (Overconsolidated Clay)}

For this soil state, the cavity expansion model should have a constitutive relationship that accurately captures undrained cohesive soil behavior. Researchers have addressed this soil state with varying success (Mayne 1991; Collins \& Yu 1996). Cao et al. (2001) and Chang et al. (2001) published companion papers that developed a closed form modified Cam clay cavity expansion model that can be used to predict tip resistance. These papers were bolstered by discussions from Ladanyi (2002) and Mayne et al. (2002). This spherical cavity model is shown in its simplified form below

$$
q_{c}=\frac{4}{3} \cdot \alpha_{\varepsilon} \cdot s_{u} \cdot\left[\ln \left(\frac{G}{s_{u}}\right)+1\right]+\sigma_{m}
$$

In Eq. (4) $q_{c}=$ tip resistance; $\alpha_{\varepsilon}=$ strain rate factor (1.64 for $10 \mathrm{~cm}^{2}$ cones); $s_{u}=$ undrained strength; $G=$ elastic shear modulus; and $\sigma_{m}=$ mean total stress. In Cao et al. (2001), the cavity limit pressure equation, the basis for Eq. (4), was compared with the solution proposed by Yu et al. (1996). Cao et al. (2001) used a small strain assumption in the derivation of their closed form solution, whereas Collins and Yu (1996) used a large strain assumption for their numerically generated solution. The two methods compare favorably. The predictive form of this cavity expansion model [Eq. (4)] was compared to both laboratory and field data by Chang et al. (2001) and shown to agree reasonably well.

The Cao et al. (2001) cavity model results indicate that OCR has only a small effect on predicted tip resistance. Yu et al. (2000) included a preliminary analysis of overconsolidated soils using their steady state model and also found that OCR had little effect on the predicted tip resistance. Based on the trends observed in the normally consolidated cohesive soils it is hypothesized that overconsolidated soils behave in a similar fashion, and that tip and sleeve normalize equivalently.

To confirm this, field data of young bay mud from the San Francisco Bay was used to calculate normalization exponents for the tip and sleeve. The young bay mud is slightly overconsolidated $(\mathrm{OCR} \cong 1.2)$ and deposited in relatively homogenous layers of sufficient thickness to provide the response of tip and sleeve resistance over a large range of overburden pressures. These results indicate an equivalent normalization exponent for the tip and sleeve (Figs. 3 and 4). The normalization exponent range for this soil state (cohesive overconsolidated) is shown in the lower right corner of Fig. 4. 


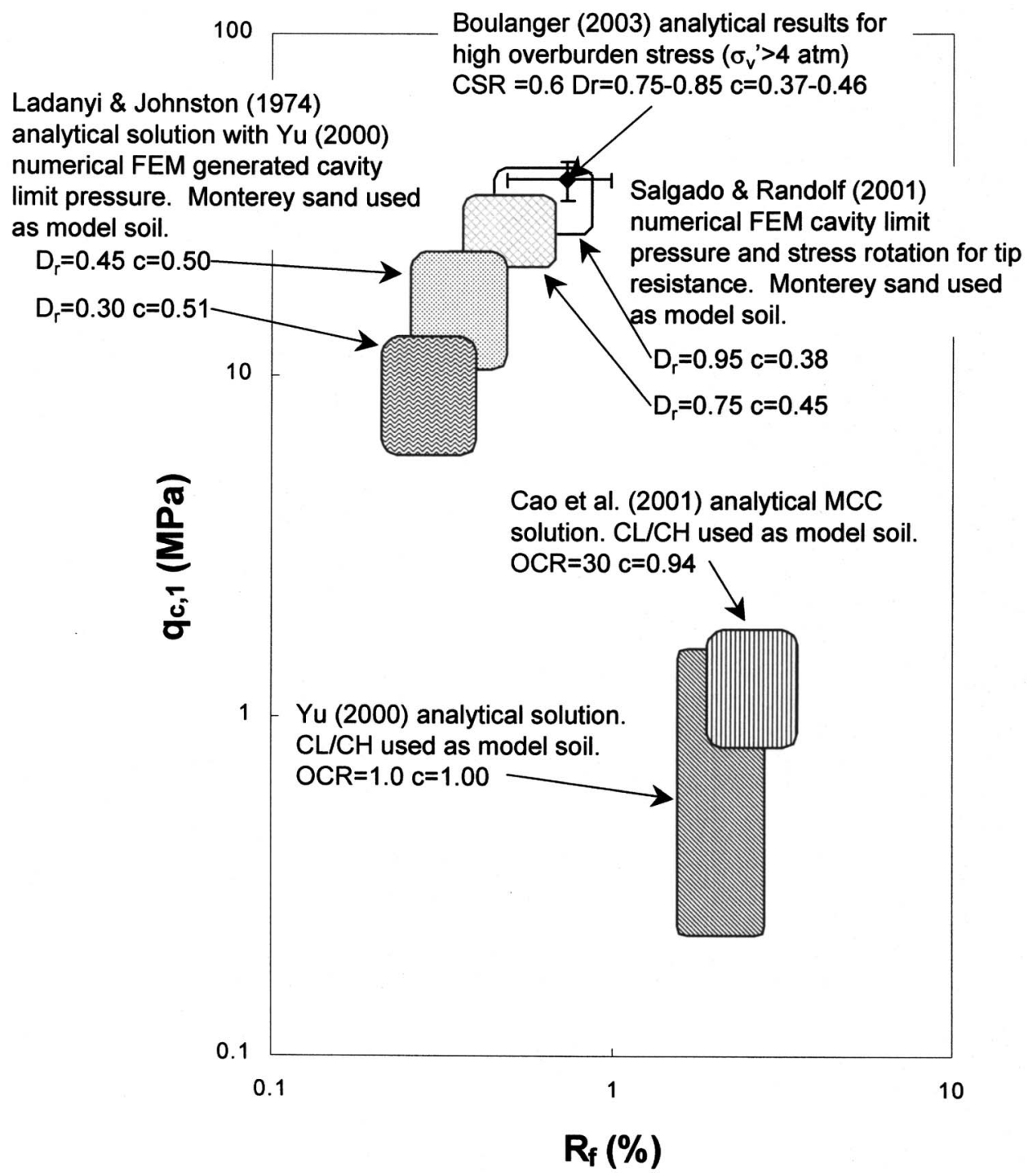

Fig. 5. Tip normalization exponent results from cavity expansion analyses

\section{Cohesionless Contractive (Loose Sand)}

Ladanyi and Johnston (1974) derived an analytical solution for tip resistance in contractive sands using a spherical cavity approach and linear elastic-plastic von Mises failure criteria

$$
q_{c}^{\prime}=N_{q} \cdot \sigma_{v 0}^{\prime}
$$

where

$$
N_{q}=\frac{\left(1+2 K_{0}\right) A}{3}\left[1+\sqrt{3} \tan \left(\lambda \phi^{\prime}\right)\right]
$$

In Eq. (5) $q_{c}^{\prime}=$ tip resistance; $N_{q}=$ cone factor; $\sigma_{v 0}^{\prime}=$ vertical effective stress; $K_{0}=$ coefficient of lateral earth pressure; $A=$ ratio of effective spherical cavity limit pressure to initial mean effective stress; $\lambda=$ cone roughness factor $(0$ for perfectly smooth and 1 for perfectly rough); and $\phi^{\prime}=$ effective friction angle. $A=$ function of strength and stiffness in which no closed-form solution exists, therefore it is calculated numerically. Yu (2000) developed a numerical solution, implemented in the code CAVEXP, for calculating the spherical cavity limit pressures needed to determine $A$. The combination of Yu's numerical solution for the limit pressure and Ladanyi and Jonhston's analytical solution for the relation between the limit pressure and tip resistance compares quite favorably with empirical results (Yu and Mitchell 1998).

The combination of the Ladanyi and Johnston's (1974) analytical solution and the Yu's (2000) numerical solution for limit pressures was calibrated using chamber test data of various sands from Salgado-Rodrigues (1993) exhaustive compendium of chamber test results. The calibrated model was then used to predict tip resistance and tip normalization exponents.

As with the cohesive soils, there is currently no analytical solution for sleeve resistance in cohesionless soils. In theory the sleeve resistance, $f_{s}$, is a function of $\sigma_{h}^{\prime}$ the horizontal effective stress and $\phi^{\prime}$ the effective friction angle

$$
f_{s} \propto \sigma_{h}^{\prime} \cdot \tan \phi^{\prime}
$$

However, sleeve resistance is assumed to be theoretically based on a cylindrical cavity expansion geometry versus a spherical geometry for the tip resistance in granular materials. This difference between tip and sleeve resistance is a function of the ratio of limit pressures for a cylinder versus a sphere $\left(1: \pi h^{2}\right)$. Collins et al. (1994) included a brief assessment of the differences of cylindrical versus spherical cavity geometries derived for sands. The results indicate that there is a divergence of the tip and sleeve 
exponent for the contractive soil state in granular materials.

Chamber tests were used to calibrate a form of Eq. (6) and approximate sleeve resistance values were correlated with the predicted tip resistance values. The normalization exponent range for this soil state (cohesionless contractive) is shown in the upper left corner of Fig. 5.

\section{Cohesionless Dilatant (Dense Sand)}

Salgado-Rodrigues (1993) developed a nonlinear elastic-plastic cavity expansion model that accounts for dilatant behavior in granular material. This model requires a finite element solution for the cavity limit pressure, which has been implemented in the code CONPOINT (Salgado et al. 1997b; Salgado and Randolf 2001). For this soil state, Salgado's model first numerically calculates the cylindrical cavity limit pressure, then uses a stress rotation analysis to obtain the tip resistance. Salgado's model follows the equation

$$
q_{c}=2 p_{L} e^{\pi \tan \phi T} \frac{(1+C)^{1+\beta}-(1+\beta) C-1}{C^{2} \beta(1+\beta)}
$$

In Eq. (7) $q_{c}=$ tip resistance; $p_{L}=$ limit cylindrical cavity pressure; $\phi_{T}=$ friction angle near the tip; and $C$ and $\beta=$ equation parameters described in Salgado et al. (1997b). Yu and Mitchell (1998) have shown that this model agrees well with a large body of empirical data. The latest version of this model, Salgado and Randolf (2001), has been used for this study. The normalization exponent range for this soil state (cohesionless dilatant) is shown in the upper left corner of Fig. 5.

Boulanger (2003) used Salgado's model as a theoretical basis to expand the range of normalization exponents for materials subjected to high overburden stresses $\left(\sigma_{v}^{\prime}>4 \mathrm{~atm}\right)$ and undergoing cyclic loads. Chamber test results from Salgado-Rodrigues (1993), for samples of $D_{r}=0.75-0.85$ and $\sigma_{v}^{\prime}>4$ atm (as corresponding to Boulanger's model) were used to locate the normalization exponent range in $R_{f}$ versus $q_{c, 1}$ space with the other cavity expansion model results. There is good agreement with Boulanger's analysis and the other models used in this study, as can be seen in Fig. 5 .

Keaveny and Mitchell (1988) took an approach that returned to the seminal work in analytical cavity expansion solutions by Vesic (1972). Their results indicate that for soils of high compressibility (low $I_{\text {rr }}$ ), a spherical cavity model predicted tip resistance best. This agrees with the cohesionless contractive results using Ladanyi and Johnston's (1974) closed form solution with Yu's (2000) numerical cavity limit pressure code. The corollary state is a low compressibility soil (high $I_{\text {rr }}$ ), which was shown to agree best with a cylindrical cavity model.

Again, assuming that the sleeve resistance is based on cylindrical cavity expansion, the above results would indicate that there is minimal divergence of the tip and sleeve normalization exponent for dilatant granular soils. However, it is believed that dilatant soils will tend to arch at the trailing edge of the cone tip. This can lead to a higher horizontal effective stress for the tip than for the sleeve and would suggest a divergence of tip and sleeve normalization exponents with increased dilatancy.

\section{Cavity Expansion Results}

The results from the cavity expansion analyses are presented in Fig. 5 as a plot of the calculated tip normalization exponents over their respective $q_{c, 1}$ and $R_{f}$ ranges. The theoretical model results were generated for an effective stress range of 0.5-3.0 atm, except for results from Boulanger's (2003) model for effective stress values higher than $4.0 \mathrm{~atm}$.

The results from the theoretical analyses (Fig. 5) were superimposed over the empirical results from Olsen and Mitchell (1995) (Fig. 1). Offshore data in Olsen and Mitchell (1995) required a slight modification due to a previous incorrect assessment of the in situ effective stress conditions (Olsen, private communication 2003). Olsen and Mitchell (1995) originally fit the empirical data using a curve fitting technique that accounted for bias error. The new normalization exponent curves (Fig. 6) were arrived at by judgmentally adjusting the previous empirically based curves with respect to the new theoretical results in Fig. 5. The disparate nature of the empirical and theoretical data did not lend to a more rigorous curve fitting procedure.

Comparison of Olsen and Mitchell (1995) with the new curves indicates that the largest change occurs in the cohesive normally consolidated region (low $q_{c, 1}$, high $R_{f}$ ) and the cohesionless contractive region (high $q_{c, 1}$, low $R_{f}$ ). The change in the cohesive normally consolidated region can be attributed mainly to the

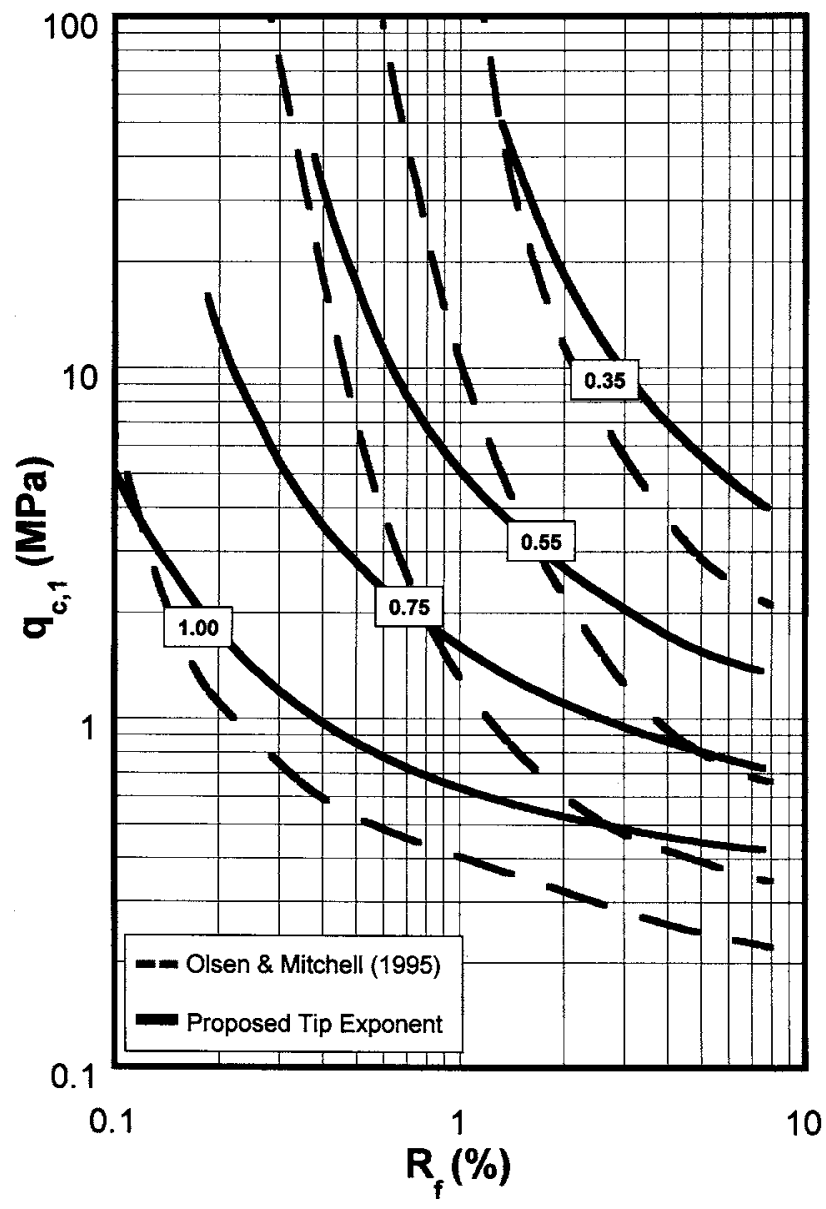

Fig. 6. Comparison of proposed tip normalization exponent curves versus Olsen and Mitchell (1995) 
correction needed in the effective stress of the offshore data from Olsen and Mitchell (1995). The trend and magnitude of this correction was confirmed by the cavity expansion results. The change in the cohesionless contractive region can be attributed as being mainly due to the difficulty of creating loose sand samples in a calibration chamber test. This was also confirmed by cavity expansion analyses.

\section{Lateral Stress Effects}

Thus far, this study has presented the effects of overburden stress on CPT measurements assuming "at rest" lateral stress conditions, or a coefficient of lateral earth pressure $\left(K_{0}\right)$ of $1 / 2$. This assumption is considered valid for recent soil deposits that have not experienced significant changes in stress conditions since deposition. It is also a reasonable assumption because it reflects the most common in situ stress condition that geotechnical engineers encounter.

As stated previously, cohesionless soils respond to overburden stress primarily as a function of relative density $\left(D_{r}\right)$ and the coefficient of lateral earth pressure $\left(K_{0}\right)$, and cohesive soils respond to overburden stress primarily as a function of OCR and undrained strength $\left(s_{u}\right)$. This would indicate that lateral earth pressure is a primary variable in the determination of cone tip resistance in cohesionless soils, and a secondary variable related through the mean stress $\left(\sigma_{m}\right)$ for cohesive soils.

To evaluate the effects of lateral stress on normalization the cavity expansion models were used to calculate the changes in the normalization exponent as a function of changes in $K_{0}$. That lateral earth pressure coefficient was varied from $1 / 2$ to 1 . The changes in normalization exponents were calculated for the models and exponent regions shown in Fig. 5. For cohesive soils a change in lateral earth pressure $\left(K_{0}\right)$ alters the shear modulus through a change in the mean stress $\left(\sigma_{m}\right)$. The normalization exponent for both normally consolidated $(\mathrm{OCR} \sim 1)$ and highly overconsolidated $(\mathrm{OCR} \sim 40)$ cohesive soils increased by less than $1 \%$ with a change in $K_{0}$ from $1 / 2$ to 1 . For cohesionless contractive soils (loose sands) the change in the normalization exponent also showed less than $1 \%$ increase for relative densities less than $D_{r}=45 \%$. For cohesionless dilatant soils (dense sands) the increase in the normalization exponent was on the order of $4.5 \%$ for a relative density of $D_{r}=75 \%$, and $11.0 \%$ for a relative density of $D_{r}=95 \%$.

These results indicate that increased lateral earth pressures have little or no impact on the normalization exponent of cohesive soils, but have some impact on the normalization exponent of cohesionless soils as a function of the relative density. Therefore, given a lateral earth pressure coefficient greater than $1 / 2$, the normalization exponent for the dense sand region in Fig. 5 would be expected to be slightly higher. However, in practice measuring the in situ lateral earth pressure coefficient is notoriously difficult and unreliable. Because the impact of a higher lateral earth pressure is not significant, is found to influence primarily dense sands, and that an assumed value of $K_{0}$ is generally used in practice, lateral stress effects are not explicitly quantified in the proposed normalization curves presented in this study (Fig. 6). For the influence of $K_{0}$ on liquefaction studies, readers are directed toward Salgado et al. (1997a,) which explores the implications of lateral stresses higher than "at rest" conditions on liquefaction resistance correlations.

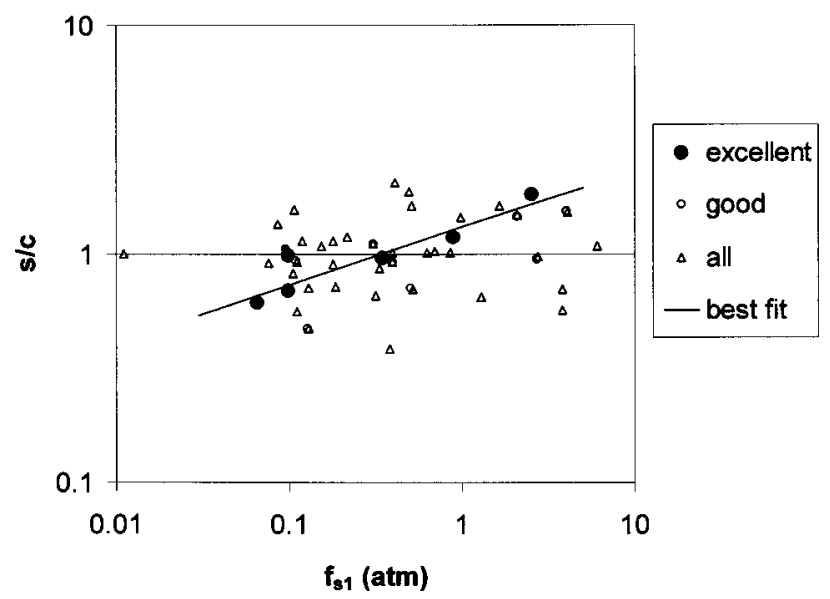

Fig. 7. Ratio of sleeve to tip normalization exponents versus normalized sleeve resistance (after Olsen 1994). Data quality assessed in Olsen (1994).

\section{Sleeve Normalization}

As discussed earlier the evaluation of sleeve normalization presents a more elusive problem because no analytical or numerical solutions for predicting sleeve resistance exist. The sleeve predictions presented so far have been based on rough assumptions in order to approximate sleeve resistance that corresponds to

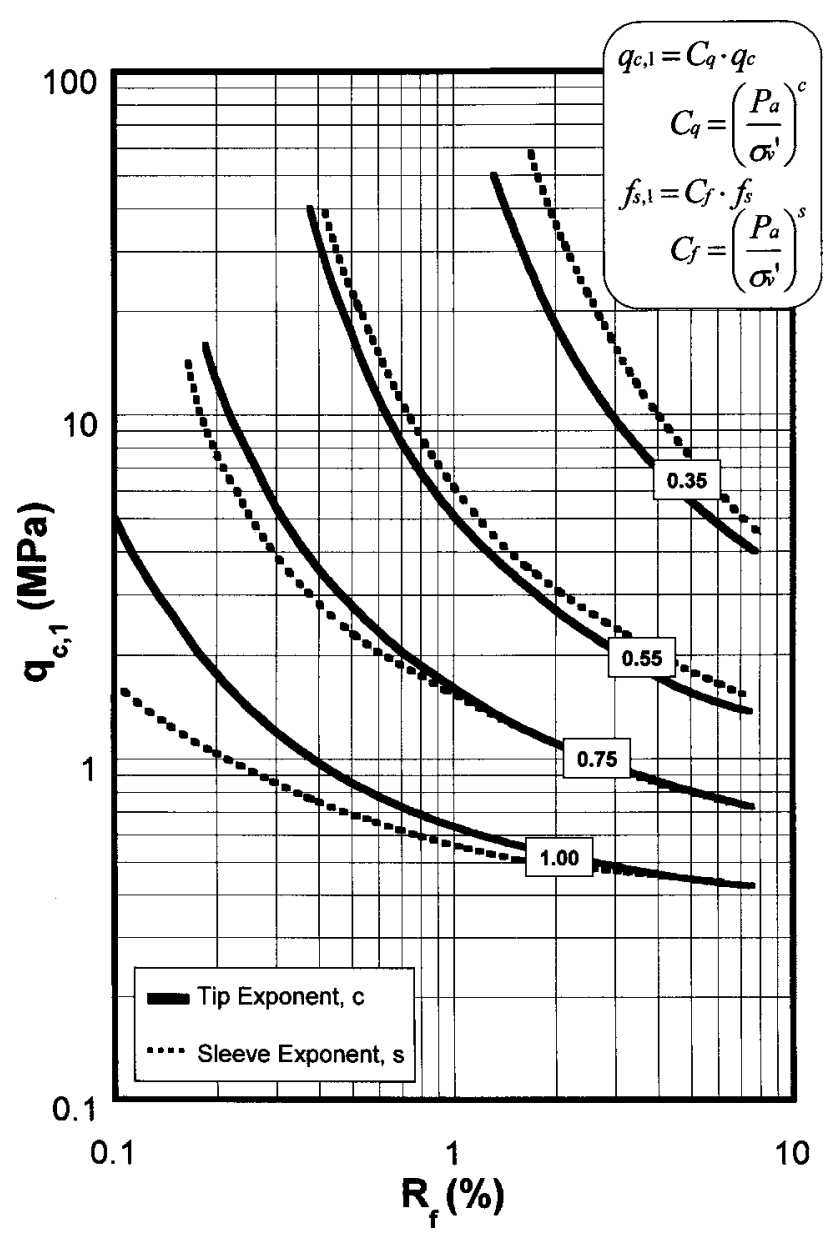

Fig. 8. Proposed tip and sleeve normalization exponent curves 
the theoretically derived tip resistance. These approximations have been calibrated using chamber data but lack the consistent and rigorous mathematical treatment that tip resistance has received.

Olsen (1994) in his compilation of field and laboratory data evaluated normalization exponents for sleeve resistance. Fig. 7 shows the results of Olsen's analysis, with the ratio of sleeve and tip normalization exponents versus normalized sleeve resistance. Olsen presented the data in this manner because it provided the strongest correlation. The trend shows the nonlinear relationship between tip and sleeve normalization exponents, but the scatter of the data is rather large. Based on the lack of conclusive results, Olsen and Mitchell (1995) recommended equivalent tip and sleeve exponents $(s=c)$, which for most applications only slightly alters the results.

From the qualitative assessment of sleeve response for different soil states presented thus far, and the data from Olsen's work, a preliminary representation of sleeve exponent curves, in relation to tip exponent curves, is presented in Fig. 8. The two sets of curves diverge in certain regions of the chart. When normalizing the tip and sleeve resistance for use in soil characterization or other engineering applications, however, the difference between $c$ and $s$ becomes insignificant. For example, let us use raw cone measurements of $q_{c}=30 \mathrm{MPa}$ and $f_{s}=300 \mathrm{kPa}$ at $3 \mathrm{~atm}$ of

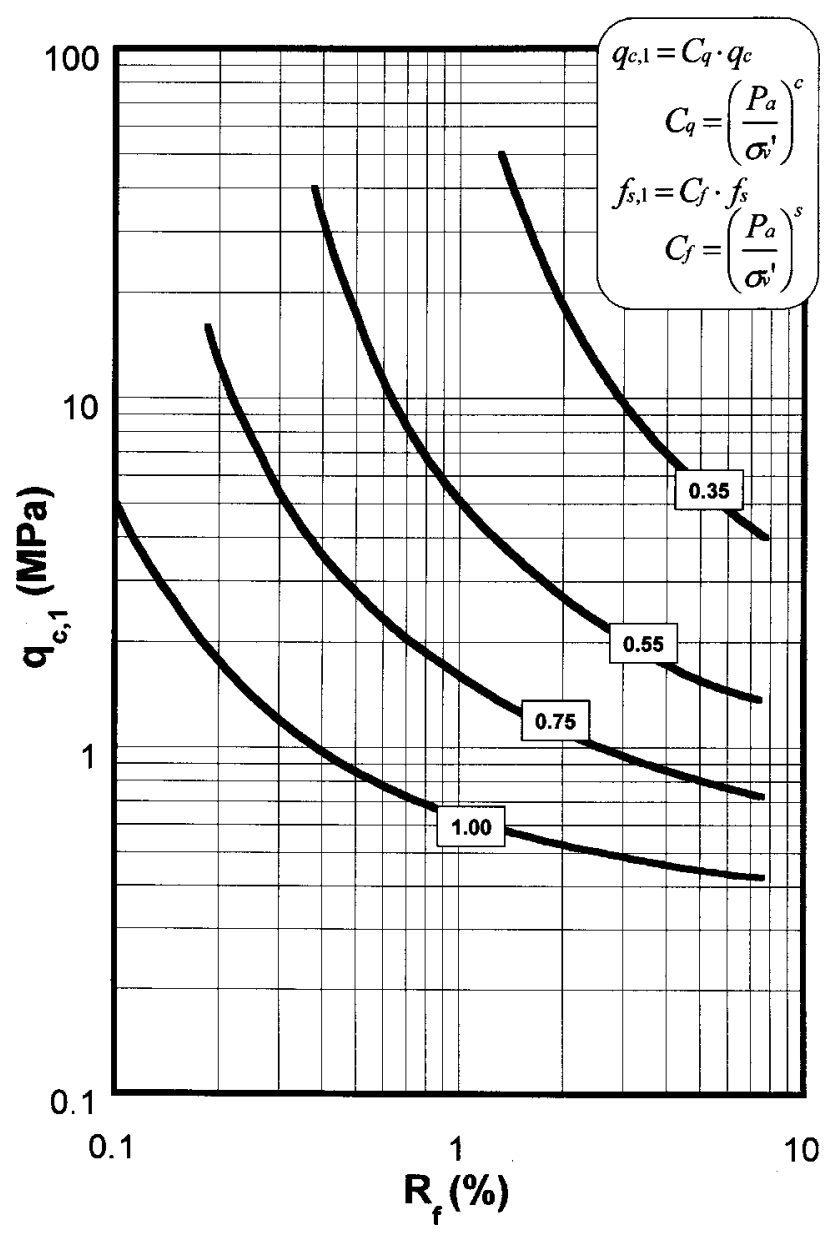

Fig. 9. Proposed tip normalization exponent curves. Eq. (8) can be used to calculate normalization exponent, $c$, in iterative normalization procedure. It is recommended that tip and sleeve resistance be normalized equivalently $(s=c)$. effective overburden, where the value of the friction ratio is $R_{f}=1.0$. (Note that these values lie in the region of Fig. 8 where a large divergence of tip and sleeve resistance is thought to occur.) Now if we take $c=0.35$ and $s=0.40$ from the curves and normalize using Eq. (1) to get $q_{c, 1}=19.7$ and $f_{s, 1}=185.4$, this gives an $R_{f}=0.942$. The overall change in $R_{f}$ is less than $6 \%$, which can be considered insignificant for most purposes. Therefore, using equivalent normalization exponents for both the sleeve and tip $(s=c)$ is a reasonable approach.

\section{CPT Normalization Procedure}

New normalization curves, as shown in Fig. 9, have been developed using current theoretical techniques and existing empirical data. To normalize the tip and sleeve appropriately, an iterative procedure is necessary. The iterative procedure involves the following steps:

1. The raw tip and friction ratio are plotted in Fig. 9 to find an initial estimate of the normalization exponent;

2. The tip is then normalized using this exponent (note: the friction ratio will not change when tip and sleeve are normalized equivalently);

3. The normalized tip resistance and friction ratio values are then replotted in Fig. 9 and reevaluated for the proximity to the initial normalization exponent value; and

4. An updated normalization exponent is selected, and the procedure repeated.

This process usually requires only two iterations to converge. To aid in computation, the following equation can be used to estimate the tip normalization exponent curves:

$$
c=f 1 \cdot\left(\frac{R f}{f 3}\right)^{f 2}
$$

where

$$
\begin{gathered}
f 1=x 1 \cdot q c^{x 2} \\
f 2=-\left(y 1 \cdot q c^{y 2}+y 3\right) \\
f 3=\operatorname{abs}[\log (10+q c)]^{z 1}
\end{gathered}
$$

and $x 1=0.78, \quad x 2=-0.33, \quad y 1=-0.32, \quad y 2=-0.35, \quad y 3=0.49$, $z 1=1.21$. In Eq. (8) $c=$ tip normalization exponent; $q_{c}=$ tip resistance; $R_{f}=$ friction ratio, and $f_{1}, f_{2}, f_{3}, x_{1}, x_{2}, y_{1}, y_{1}, y_{3}$, and $z_{1}=$ parameters of the equation. This equation has no physical significance other than it gives a good approximation of the tip normalization curves. The functional form and coefficients of Eq. (8) were found by curve fitting in log-log space. The "best" fit was chosen that minimized variance along each contour and minimized variance over the entire contour surface.

\section{CPT versus SPT Normalization}

Variable normalization for overburden stress effects on CPT measurements has been shown to be part and parcel of the in situ measuring process. The normalization exponent is an indicator of the soil state under the given stress conditions. Use of a constant normalization exponent can lead to incorrectly normalized values.

This error has also become evident in the normalization of SPT measurements where the exponent is generally taken as a constant for all soil types and stress conditions (usually 0.5). 
Researchers have noted this inaccuracy in field data and chamber test results, and have partially compensated for it by using the additional correction factor of $K_{\sigma}$ (Seed et al. 1983; Youd et al. 2001). Use of variable normalization exponents with the CPT obviates the need for $K_{\sigma}$ corrections.

Currently there are no theoretical solutions for the standard penetration test (SPT). This relegates critical analysis to chamber studies and field results. As the SPT tends to be a "nonstandard" test, the variability of the results from different researchers and different equipment has the tendency to reduce the accuracy of the data below a level needed for assessing overburden stress effects. If CPT and SPT are performed side by side in field tests, it is recommended that the normalization exponents used for the CPT also be used to normalize the SPT.

\section{Summary and Conclusions}

This paper presents the results of a comprehensive study of the normalization of CPT measurements for effective overburden stress. A review of previous field and calibration chamber test results was conducted. A theoretical framework using advances in cavity expansion analysis and steady state methods is presented. This framework gives greater confidence in a variable normalization procedure and the accuracy of normalized CPT resistance values.

New normalization exponent curves for tip and sleeve resistance are shown in Figs. 8 and 9. Because of the lack of a theoretical foundation for sleeve normalization and the small difference between the normalized results, it is recommended that the tip and sleeve be normalized equivalently $(s=c)$ using the proposed tip normalization curves. An iterative procedure is needed to converge on the appropriate normalization exponent, where convergence can usually be achieved in as little as two iterations. An equation approximating the tip normalization curves [Eq. (8)] is provided for ease of calculation.

This research was driven by the need for an improved normalization procedure for liquefaction triggering analysis. Liquefaction triggering procedures have been greatly enhanced by appropriate normalization of CPT measurements, however the results presented herein are applicable to all studies where the CPT is used.

\section{Acknowledgments}

Financial support was provided by the California Department of Transportation (CalTrans), the California Energy Commission (CEC), and Pacific Gas and Electric Company (PG\&E) through the Pacific Earthquake Engineering Research (PEER) Center's Lifelines Program, Task 3D02. This support is greatly appreciated. The writers would like to thank the anonymous reviewers for their insightful comments and suggestions that improved the content of this paper.

\section{Notation}

The following symbols are used in this paper:

$A=$ ratio of effective spherical cavity limit pressure to initial mean effective stress;

$C$ and $\beta=$ equation parameters for dilatant cavity expansion model;

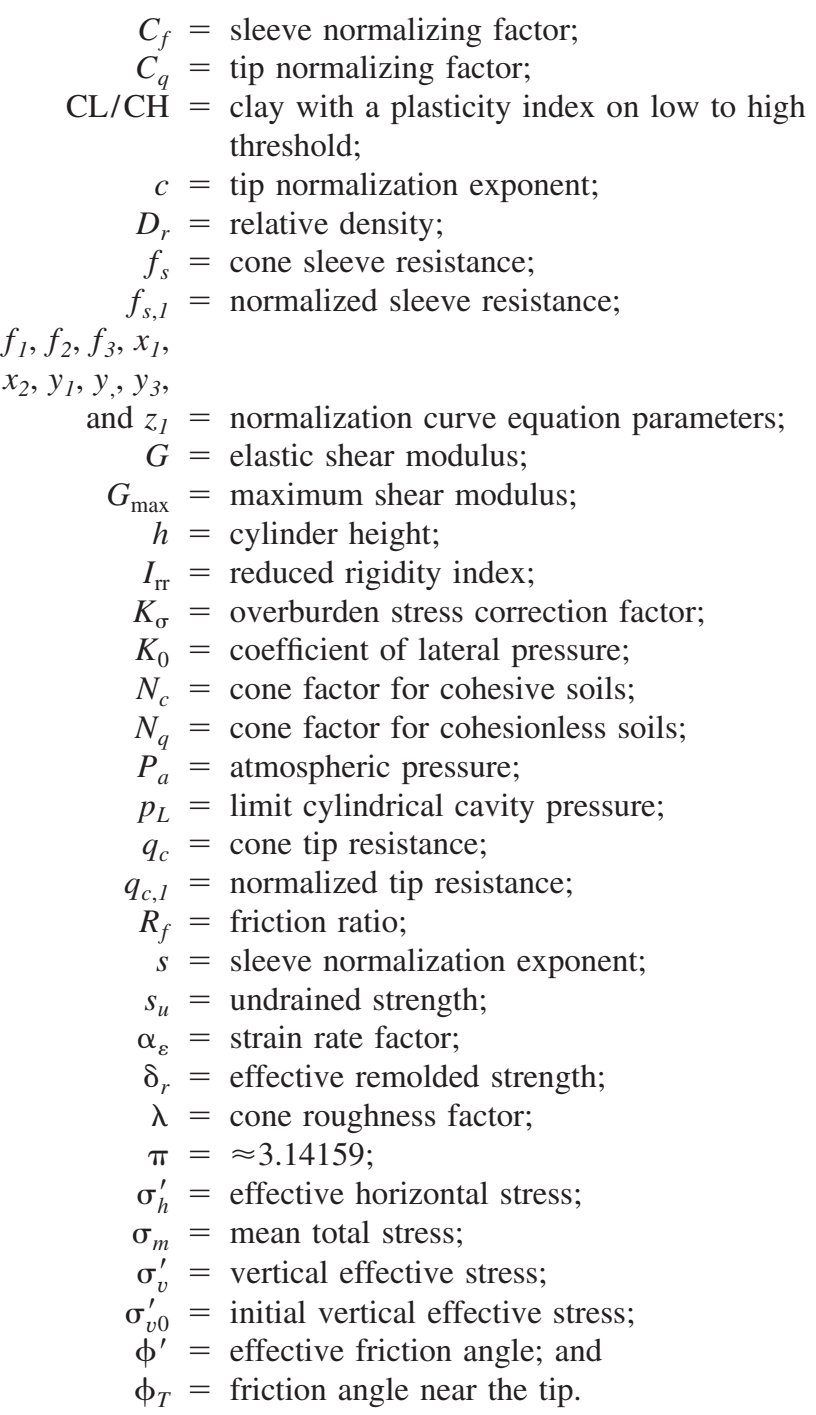

\section{References}

Baligh, M. M. (1976). "Cavity expansion in sands with curved envelopes.” J. Geotech. Eng., 102(11), 1131-1146.

Bishop, R. F., Hill, R., and Mott, N. F. (1945). "The Theory of Indentation and Hardness Tests." Proc. Phys. Soc. London, 57, 147-159.

Boulanger, R. W. (2003). "High overburden stress effects in liquefaction analyses." J. Geotech. Geoenviron. Eng., 129(12), 1071-1082.

Cao, L. F., Teh, C. I., and Chang, M. F. (2001). "Undrained cavity expansion in modified cam clay I: Theoretical analysis." Geotechnique, 51(4), 323-334.

Carter, J. P., Booker, J. R., and Yeung, S. K. (1986). "Cavity expansion in cohesive frictional soils." Geotechnique, 36(3), 349-353.

Chang, M. F., Teh, C. I., and Cao, L. F. (2001). "Undrained cavity expansion in modified cam clay II: Application to the interpretation of the piezocone test." Geotechnique, 51(4), 335-350.

Collins, I. F., Pender, M. J., and Wang, Y. (1992). "Cavity expansion in sands under drained loading conditions." Int. J. Numer. Analyt. Meth. Geomech., 16, 3-23.

Collins, I. F., Pender, M. J., and Wang, Y. (1994). "Critical state models and the interpretation of penetrometer tests." Computer methods and advances in geomechanics, Siriwardane and Zaman, eds., Balkema, Rotterdam, The Netherlands, 1725-1730.

Collins, I. F., and Yu, H. S. (1996). "Undrained cavity expansion in critical state soils." Int. J. Numer. Analyt. Meth. Geomech., 20, 489-516. 
Hardin, B. O. (1978). "The nature of stress-strain behavior for Soils." Conf. Proc., Earthquake Engineering and Soil Dynamics, Vol. 1., ASCE, New York, 3-90.

Huang, A., and Ma, M. Y. (1994). "An analytical study of cone penetration tests in granular material." Can. Geotech. J., 31(1), 91-103.

Keaveny, J. M. (1985). "In-situ determination of drained and undrained soil strength using the cone penetration test." $\mathrm{PhD}$ dissertation, Univ. of California at Berkeley, Berkeley, Calif.

Keaveny, J. M., and Mitchell, J. K. (1988). "Strength of fine-grained soils using the piezocone." Publication No.171, Norwegian Geotechnical Institute, Norway, 1-9.

Kurup, P. U., Voyiadjis, G. Z., and Tumay, M. T. (1994). "Calibration chamber studies of piezocone test in cohesive soils." J. Geotech. Eng., 120(1), 81-107.

Ladanyi, B., (2002). "Discussion of Undrained cavity expansion in modified cam clay II: Application to the interpretation of the piezocone test Chang et al. (2001)." Geotechnique, 52(4), 307-311.

Ladanyi, B., and Johnston, G. H. (1974). "Behavior of circular footings and plate anchors embedded in permafrost." Can. Geotech. J., 11, 531-553.

Masood, T. (1990). "Determination of lateral earth pressure in soils by in-situ methods." PhD dissertation, Univ. of California at Berkeley, Berkeley, Calif.

Masood, T., and Mitchell, J. K. (1993). "Estimation of in-situ lateral stresses in soils by cone penetration test." J. Geotech. Eng., 119(10), 1624-1639.

Mayne, P. W. (1991). "Determination of OCR in clays by piezocone tests using cavity expansion and critical state concepts." Soils Found., $31(2), 65-76$.

Mayne, P. W., Chen, B. S. Y., and Burns, S. E. (2002). "Discussion of undrained cavity expansion in modified cam clay II: Application to the interpretation of the piezocone test Chang et al. (2001)." Geotechnique, 52(4).

Moss, R. E. S., Seed, R. B., Kayen, R. E., Stewart, J. P., and Tokimatsu, K. (2005) "Probabilistic liquefaction triggering based on the cone penetration test." GeoFrontiers 2005, E. M. Rathje, ed.,

Olsen, R. S. (1994). "Normalization and prediction of geotechnical properties using the cone penetration test (CPT)." $\mathrm{PhD}$ dissertation, Univ. of California at Berkeley, Berkeley, Calif.

Olsen, R. S. and Koester, J. P. (1995). "Prediction of Liquefaction Resistance using the CPT." Proc. Int. Symp. on Cone Penetration
Testing, CPT 95, Linkoping, Sweden, 251-256.

Olsen, R. S., and Malone, P. G. (1988). "Soil classification and site characterization using the cone penetrometer test." Penetration Testing 1988, Proc., 1st Int. Symp. on Penetration Testing, ISOPT-1, J. A. A. De Ruiter, ed., Balkema, Orlando, Fla., 887-893.

Olsen, R. S. and Mitchell, J. K. (1995). "CPT stress normalization and prediction of soil classification." Proc., Int. Symp. on Cone Penetration Testing, CPT 95, Linkoping, Sweden, 257-262.

Robertson, P. K., and Wride, C. E. (1998). "Evaluating cyclic liquefaction potential using the cone penetration test." Can. Geotech. J., 35(3), 442-459.

Salgado, R., Boulanger, R. W., and Mitchell, J. K. (1997a). "Lateral stress effects on CPT liquefaction resistance correlations." J. Geotech. Geoenviron. Eng., 123(8), 726-735.

Salgado, R., Mitchell, J. K., and Jamiolkowski, M. (1997b). "Cavity expansion and penetration resistance in sand." J. Geotech. Geoenviron. Eng., 123(4), 344-354.

Salgado, R., and Randolph, M. F. (2001). "Analysis of cavity expansion in sand." Int. J. Geomech., 1(2), 175-192.

Salgado-Rodrigues, R. (1993). "Analysis of penetration resistance in sands." PhD dissertation, Univ. of California at Berkeley, Berkeley, Calif.

Seed, H. B., Idriss, I. M., and Arango, I. (1983). "Evaluation of liquefaction potential using field performance data." J. Geotech. Eng., 109(3), 458-482.

Vesic, A. S. (1972). "Expansion of cavities in infinite soil mass." J. Soil Mech. Found. Div., 98(3), 265-289.

Youd, T. L., et al. (2001). "Liquefaction resistance of soils: Summary report from the 1996 NCEER and 1998 NCEER/NSF workshops on evaluation of liquefaction resistance of soils." J. Geotech. Geoenviron. Eng., 127(10), 817-833.

Yu, H. S. (2000). Cavity expansion methods in geomechanics, Kluwer Academic, Boston.

Yu, H. S., Herrmann, L. R., and Boulanger, R. W. (2000). "Analysis of steady cone penetration in clay." J. Geotech. Geoenviron. Eng., 126(7), 594-605.

Yu, H. S., and Houlsby, G. T. (1991). "Finite cavity expansion in dilatant soils: Loading analysis." Geotechnique, 41(2), 173-183.

Yu, H. S., and Mitchell, J. K. (1998). "Analysis of cone resistance: Review of methods.” J. Geotech. Geoenviron. Eng., 124(2), 140-149.

Yu, H. S., Schnaid, F., and Collins, I. F. (1996). "Analysis of cone pressuremeter tests in sands." J. Geotech. Eng., 122(8), 623-632. 